\title{
Study of Interaction Between Operational Clock-Frequency and Energy Consumption of Microcontrollers for Wireless Sensor Applications
}

\author{
Subhawat Jayasvasti ${ }^{1}$, and Don Isarakorn ${ }^{1 *}$ \\ ${ }^{1}$ Instrumentation and Control Engineering Department, Faculty of Engineering, King Mongkut's Institute of Technology Ladkrabang, \\ Bangkok, Thailand
}

\begin{abstract}
This work focuses on the diagnosis of energy consumption of commonly used microcontrollers with various driving clock-frequency. The interaction among the driving clock-frequency, processing time and current consumption are determined to be a guidance for the design of low-power wireless sensor applications. In implementation, the microcontrollers are supposed to process the data following the operational procedures of wireless sensor application, which is programmed to solve mathematical equation with various driving clock-frequencies, and then sleep to reduce the power consumption. With a single circle procession, the current consumption at each driving clock-frequency are captured and analyzed. As the results, the current consumption is proportional to the driving clock-frequency, however energy consumption is minimized at highest clock-frequency.
\end{abstract}

\section{Introduction}

The wireless sensor applications have been ubiquitous in various field of works including sport, heath monitoring, transportation, agriculture and industrial [1-3]. With its considerable growth, the reduction in energy consumption of wireless application has been recently trendy, since the low-power device can operate with a few batteries during its lifespan. It can decrease the use of batteries and enables the realization of autonomous energy system powered by harvested ambient energy. Typically, the main strategy for decreasing the power consumption is to reduce the size of electronic components, and program the wireless devices to automatically wake up only for data measurement and then sleep after data was transmitted [4]. lower case. You should leave $35 \mathrm{~mm}$ of space above the title and $6 \mathrm{~mm}$ after the title.

Out of strategy mentioned above, one more main challenge is the appropriate speed of data procession of microcontroller composed in wireless sensor application because processing speed is proportional to the current consumption and clock-frequency driving the microcontroller. For example, Figure. 1 demonstrates the interaction between clock-frequency and current consumption of microcontroller (ATMega32) [5]. As can be seen, the high clock-frequency resulting high current consumption, while low current consumption is observed at low clock-frequency. However, these current consumptions

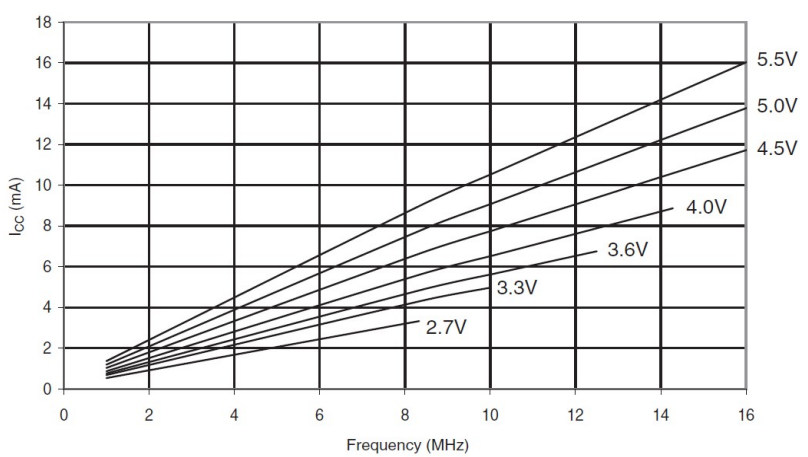

Fig. 1. The interaction between clock-frequency and current consumption.

The cannot obviously demonstrate the optimal clock-frequency resulting in low energy consumption, since the energy consumption derive from the power consumption and time interval of data procession of microcontroller. With a same dataset procession, the high processing speed take a short time for data procession, but the high-power consumption is required. On the other hand, the low processing speeds can operate with the low-power consumption, but it takes a long processing time [6].

Therefore, purpose of this paper is to find out the optimal clock-frequency, and microcontroller consuming the lowest energy among the commonly used microcontrollers for further design of low-power wireless sensor application. Firstly, the investigation method is designed following the operational feature of

* Don Isarakorn: don.is@kmitl.ac.th 
wireless sensor application. Then, the experiment is implemented. After that, the experimental results are discussed. And, finally the conclusion is given.

\section{Investigation Method}

To achieve the reasonable results of investigation, the interaction between clock-frequency and energy consumption of microcontrollers is considered following the working sequence of wireless sensor application in a working circle: data measurement, procession, communication and sleep as shown in Fig. 2. The clockfrequency driving microcontrollers is increased from 1 $\mathrm{MHz}$ to the maximum frequency. The current consumptions at each driving clock frequency are captured by using digital multi-meter (Agilent 34411A) [7]. After that, the experimental results are analyzed and utilized to find out the microcontroller consuming the lowest-energy. The microcontrollers investigated in this work are shown in Fig. 3 and listed in Table 1.

\section{Experimental Design}

Following the section 2, the experiment can be designed into five cases as below:

First, the microcontrollers are programmed to process the inconstant pointer changing the pointing location with a delay function (delay ()) in an infinite while loop (while (1)) for considering the current consumption at various driving clock frequency.

Second is the computation of a complex mathematical equation taking a bit long processing time, which supposes the procession of data derived from sensor. In the implementation, the microcontrollers are used to calculate the 2000 digits of $\mathrm{Pi}$ following a Nilakantha series as below:

$$
\pi=3+4 \sum_{k=0}^{\infty} \frac{-1^{k}}{(2 k+2)(2 k+3)(2 k+4)}
$$

where, $k$ is the number of digits.

And, the answer is stored in a long double variable (64 bits). Then, the processing time with each driving clock-frequency is observed.

Third, an equation (1) is processed in loop function to find out the average current consumption at each clock frequency.

Fourth is to examine the current consumption of microcontrollers in energy saving mode. This case assumes that the wireless sensor is stayed in sleeping or power-down state.

And finally, the microcontrollers are supposed to process in one operating circle of wireless sensor node as calculating the 2000 digits of $\mathrm{Pi}$ and sleeping for 5 seconds. Then, by adjusting the clock frequency, the current consumption and time interval of procession are analyzed for considering the energy consumption [8].

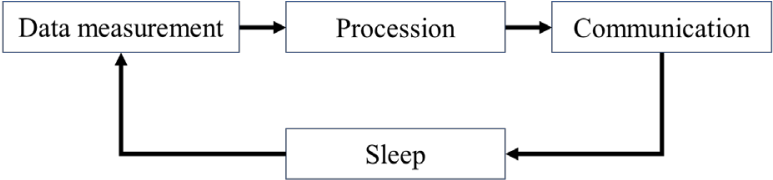

Fig. 2. Working circle of wireless sensor application.

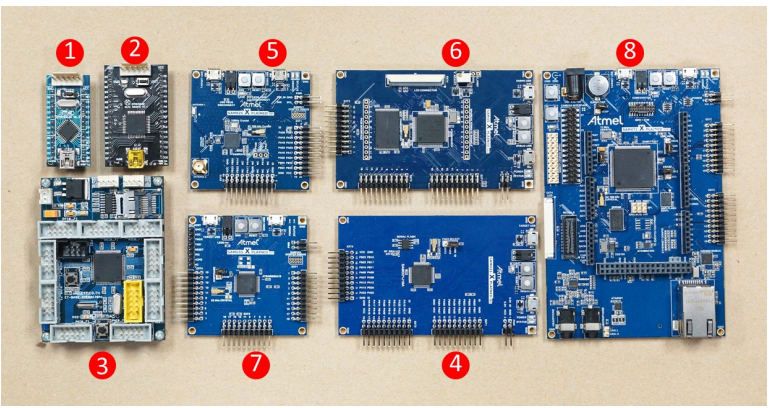

Fig. 3. The complete microcontroller boards

Table 1. The investigated microcontrollers.

\begin{tabular}{|c|l|c|}
\hline No. & \multicolumn{1}{|c|}{ Microcontrollers } & $\begin{array}{c}\text { Maximum clock } \\
\text { frequency } \\
\text { (MHz) }\end{array}$ \\
\hline 1 & ATmega168 AVR/8bit & 16 \\
\hline 2 & ATmega32 AVR/8bit & 16 \\
\hline 3 & ATXmega128A1 AVR/16bit & 32 \\
\hline 4 & ATSAMD21J ARM Cortex M0/32bit & 48 \\
\hline 5 & ATSAMR21G ARM Cortex M0/32bit & 48 \\
\hline 6 & ATSAM4S ARM Cortex M4/32bit & 120 \\
\hline 7 & ATSAMG55 ARM Cortex M4/32bit & 120 \\
\hline 8 & ATSAMV71 ARM Cortex M7/32bit & 300 \\
\hline
\end{tabular}

\section{Results and Discussion}

From the first experiment, the current consumption resulting from the procession of inconstant pointer was shown in Fig. 4. The current consumptions were in the same trend, which were maximized at highest driving clock frequency. The microcontroller consuming the lowest current at driving clock frequency of $1 \mathrm{MHz}$ is SAMG55. The consumed current was $0.9 \mathrm{~mA}$.

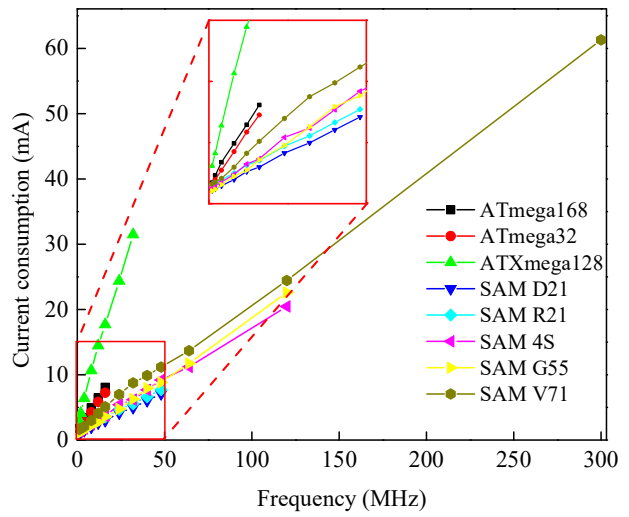

Fig. 4. Current consumption with increasing of clock frequency 
With the second experiment, the processing times taken for calculating 2000 digits of $\mathrm{Pi}$ at each driving clock frequency were shown in Fig.5. The shortest processing time is noticed at highest clock frequency. The increasing clock frequency results the data processing speed increased. On the other hand, the results also presented the mathematic calculation performance of each microcontroller. The highestperformance microcontroller spends a shortest processing time. As the results, at driving clock frequency of $1 \mathrm{MHz}$, a SAMV71 present the best performance with processing time of $455.2 \mathrm{~ms}$.

Figure. 6 show the results of third experiment. The current consumptions of microcontrollers are higher than the first experiment because the microcontrollers write and read the data into RAM. The lowest current consumption of $1.18 \mathrm{~mA}$ was found at SAMD21.

Following the fourth experiment, the current consumptions in the energy saving mode (sleeping mode) of microcontrollers were found as show in the Table II. As the results, the microcontrollers consume the different amount of energy due to their architectures. The ATSAMR21 and ATSAMV71 microcontrollers consume the much higher current than others, since SAMR21 composed of the wireless module inside which still consume the current even stayed in the energy saving mode, and SAMV71 is designed to consist of the complex integrated circuit for high-performance of data procession. With very high current consumption, the ATSAMR21 and ATSAMV71 microcontrollers are not appropriate for low-power wireless application. On the other hand, the xmega 128 presented the very low current consumption which is $0.27 \mu \mathrm{A}$.

The trends of energy consumptions illustrated in Fig. 7 derived from last experiment. The energy consumptions decreased due to the increase of driving clock frequency. With maximum driving clock frequency, the lowest energy consumption of each microcontroller was found as shown in Fig. 8. As can be seen, the SAMG55 consume the lowest energy which is $1.22 \mathrm{~mJ}$.

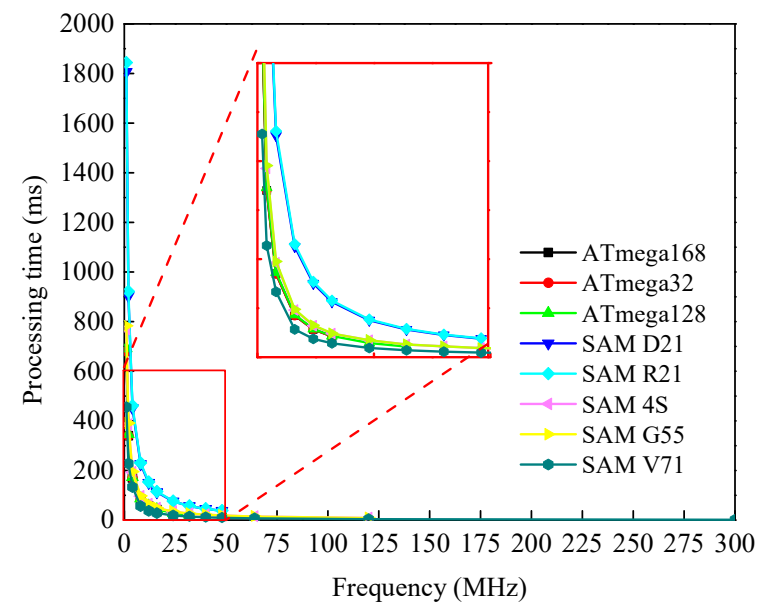

Fig. 5. Processing time for calculating the 2000 digits of Pi.
This work can be summarized that even through the high driving clock frequency is a cause of high current consumption, it results in the decrease of energy consumption. Therefore, the microcontrollers should be driven with their maximum clock frequency for wireless sensor application consuming low-energy.

Table 2. Current consumption for sleeping mode.

\begin{tabular}{|c|c|}
\hline \multicolumn{2}{|c|}{ Current consumption $(\boldsymbol{\mu A})$} \\
\hline ATMega168 & 32.00 \\
\hline ATMega32 & 41.59 \\
\hline ATXmega128 & 0.27 \\
\hline ATSAMD21 & 3.01 \\
\hline ATSAMR21 & 460.59 \\
\hline ATSAMG55 & 57.14 \\
\hline ATSAM4S & 20.45 \\
\hline ATSAMV71 & 1344.18 \\
\hline
\end{tabular}

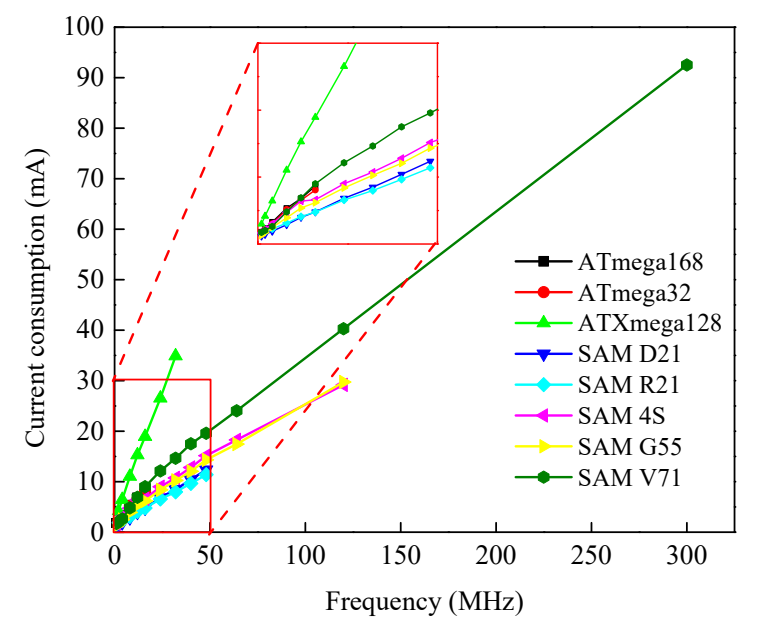

Fig. 6. Interaction between current consumption and driving clock frequency for calculating the 2000 digits of Pi. 


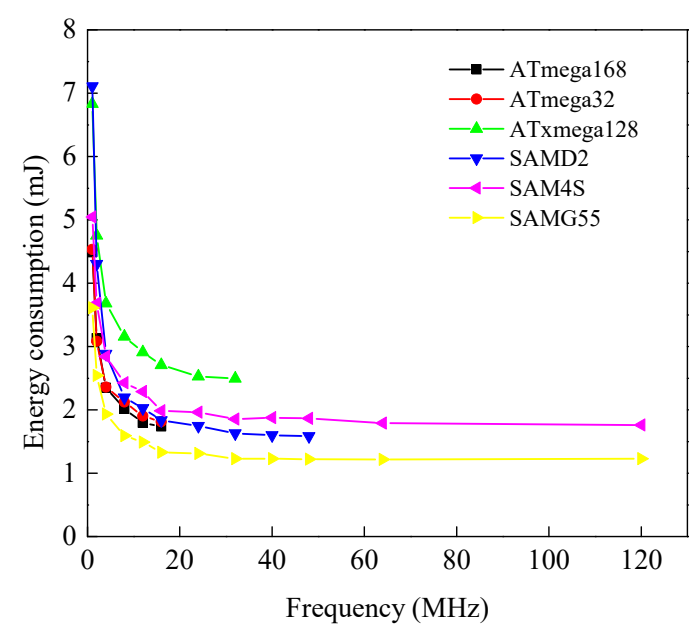

Fig. 7. Energy consumption with increasing of driving clock frequency.

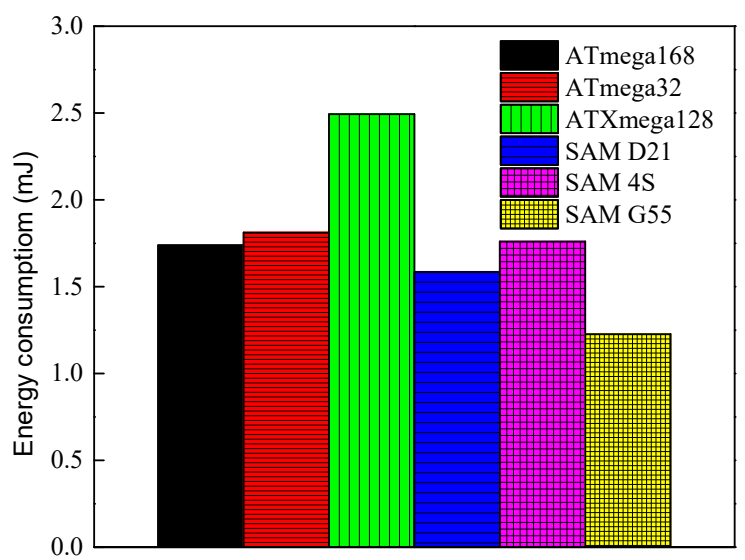

Fig. 8. The energy consumption of microcontrollers at maximum driving clock frequency.

\section{Conclusion}

This paper presented the experimental study of interaction between clock frequency and energy consumption of microcontrollers for wireless sensor application. The current energy consumptions resulted from the various driving clock frequencies were investigated with following the operational feature of wireless sensor application. As the results, the microcontrollers consumed the lowest energy at their maximum driving clock frequency.

Moreover, this work also demonstrated that the selection of microcontrollers for wireless application consuming the low energy can be considered by flowing the performances below:

- The mathematic data processing speed.

- The maximum driving clock frequency.

- The current consumption in the active and sleep modes.

\section{Acknowledgement}

This work was supported by King Mongkut's Institute of Technology Ladkrabang Research Fund [KREF016101].

\section{References}

1. T. Sudhawiyangkul, D. Isarakorn, Design and optimization of a small-scale linear electromagnetic energy harvester, pp. 1-6, (2015)

2. J. Kim ; C. Chu, Analysis of energy consumption for wearable ECG devices, pp. 962-965, (2014)

3. K. Masaba, A. Ntakirutimana, T. Ustun, Design and implementation of a smart irrigation system for improved water-energy efficiency, pp. 1-5, (2016)

4. T. Krongtripop, A performance comparisong of energy conservation between idle mode and power down mode for microcontroller in wireless mobile node, pp. $552-556,(2010)$

5. Atmel ATMega32 datasheet "Active Supply Current vs. Frequency", pp 308.

6. I. Tsekoura et al, An evaluation of energy efficient microcontrollers, pp. 1-5, (2014)

7. S. Janković, V. Drndarević, Microcontroller power consumption measurement based on PSoC, pp. 673 $-676,(2015)$

8. S. Suradhanwiar ; S. Durbha ; J. Adinarayana Energy optimization in wireless sensor networks using duty-cycling approach, pp. 1-6, (2017)

9. Microchip ATSAMV71 datasheet "Atmel-44003EATARM-SAM V71-Datasheet_12-Oct-16”, (2016). 